\title{
A laboratory apparatus to study Thermal Response Test in the presence of groundwater flow
}

\author{
Adriana Angelotti1 *, Luca Molinaroli1 \\ ${ }^{1}$ Politecnico di Milano, Energy Department, v. Lambruschini 4, 20156 Milano, Italy
}

\begin{abstract}
The standard approach to Thermal Response Test, based on conduction heat transfer in the ground, turns out to be unsuccessful under significant groundwater flow. The applicability of the Moving Infinite Line Source model to interpret the TRT in this case still needs to be proved. In order to study the TRT in the presence of a groundwater flow, an original laboratory apparatus has been developed. The Sand Box design is based on a heat transfer similitude between the real scale TRT problem and the laboratory scale one. The Sand Box sizes $(1,2 \mathrm{~m} \times 0.6 \mathrm{~m} \times 1.0 \mathrm{~m})$ are then set in order to keep the boundaries unaffected by the heat source during the TRT. The U-pipe heat exchanger is reproduced through a twocables electrical resistance $1 \mathrm{~m}$ long. A hydraulic loop with a peristaltic pump allows to obtain a Darcy velocity across the sandy soil up to $6,75 \cdot 10^{-5} \mathrm{~m} / \mathrm{s}$. The measurement system consists in several thermocouples in the porous medium and in a flow meter. The TRT results at null groundwater velocity allow to derive a reference thermal conductivity. The first tests with groundwater flow show the suitability of the apparatus and allow to derive some preliminary considerations.
\end{abstract}

\section{Introduction}

Thermal Response Test (TRT) is a well-known experimental procedure allowing to measure in situ the average ground thermal conductivity and the borehole thermal resistance, namely key parameters for the correct sizing of the borehole heat exchangers. Since the first full scale test rigs developed in Sweden and USA in the mid '90s [1], TRT has widespread worldwide [2]. The standard TRT consists in injecting/extracting a constant thermal power into the ground by circulating a given mass flow rate of carrier fluid in a pilot borehole. The recorded carrier fluid temperature variation with time is usually interpreted with the Infinite Line Source (ILS) model [3]. By solving the inverse heat transfer problem the ground and the borehole thermal properties are derived. According to the international standard [4], the test duration should be based on the convergence of the measured thermal conductivity to a constant value, typically occurring in $48-72 \mathrm{~h}$.

When a regional groundwater flow is present, the hypothesis of pure conduction in the ground fails, due to advection and even thermal dispersion contributions to heat transfer. If a TRT is performed under these circumstances and the ILS model is adopted, either an artificially high thermal conductivity is derived or it becomes impossible to estimate any [5]. Moreover, the objective of a TRT in the presence of groundwater flow should be measuring both thermal conductivity and Darcy velocity.

In the last years the Moving Line Source (MLS) model [6] has been proposed to interpret groundwaterinfluenced TRTs. Wagner et al. [7] apply the MLS to numerically simulated TRTs, showing that the actual Darcy velocity is systematically underestimated, as a consequence of the negligible hydraulic conductivity of the grouting material. Chiasson and O' Connell [8] compare the MLS, the groundwater g-functions and a mass-transport solution including thermal dispersion as analytical models for TRT analysis in the presence of groundwater flow. They find that only the mass-heat transport analogy yields a favorable comparison to field test data, implying that thermal dispersion is an important parameter, at least for relatively high groundwater velocities. By applying the MLS model to field TRTs, Angelotti et al. [9] show that the inverse problem is ill-posed and may lead to multiple solutions. Moreover, in the cases where conduction and advection compete, the MLS approach is not successful in determining the Darcy velocity in a sufficiently narrow range.

In addition, Rouleau and Gosselin [10] develop a new conceptual TRT in which heating cable sections

\footnotetext{
${ }^{*}$ Corresponding author: adriana.angelotti@polimi.it
} 
inject heat into the borehole and some temperature probes measure the temperature variation at the borehole wall at a given depth. They find that this combined hydro/thermal test is more sensitive to the ground thermal conductivity than to the groundwater velocity, the latter being derived via the differences of temperatures read by the sensors.

Therefore, further investigations are necessary to identify the suitable methodology to perform and/or analyse a groundwater-influenced TRT. Such investigations are better carried on with a laboratory apparatus, where ground properties and groundwater flow can be measured independently and varied more easily than in field. This paper describes the development of a Sand Box apparatus designed for this purpose, as well as the first tests executed through it.

\section{Sand Box development}

\subsection{Literature review on sand boxes}

In order to develop a laboratory apparatus for the study of the TRT under groundwater flow, a literature review was firstly performed, concerning sand boxes used to investigate heat transfer problems connected to ground heat exchangers. The aim was to review geometrical and physical scaling approaches, boundary conditions, materials choices, presence of groundwater flow and finally measurement points.

Beier et al. [11] construct a large wooden box with a square section with sides of $1.8 \mathrm{~m}$ and length of $18 \mathrm{~m}$. A U-pipe is inserted into a $12.6 \mathrm{~cm}$ aluminum pipe centered horizontally along the length of the box and serving as the borehole wall. The box is filled with water saturated sand. To minimise thermal interference from the surrounding room, a guarded space is created around the box where a temperature controlled airflow is circulated. A testing unit for TRT is connected to the Upipe and supplies approximately $1100 \mathrm{~W}$ to the circulating water. Several thermistors are placed inside the sand box to record temperature at the borehole wall and in the medium.

Kramer et al. [12] build a steel tank with a $1.83 \mathrm{~m} \times$ $1.83 \mathrm{~m}$ square section, divided into a lower portion 1.22 $\mathrm{m}$ tall and an upper portion $0.91 \mathrm{~m}$ high. The tank, filled with a dry sand bed, hosts a precast concrete geothermal pile (diameter $0.1 \mathrm{~m}$, length $1.38 \mathrm{~m}$ ) embedding a Ushaped pipe (outer diameter $15.8 \mathrm{~mm}$ ). Thermal performance tests are carried out at constant inlet fluid temperature. The soil temperature is measured in several points inside the sand tank. Thermal load test can be performed for about 7 days without observing any temperature change at the tank boundaries.

Katsura et al. [13] adopt an acrylic cylinder with internal diameter $300 \mathrm{~mm}$ as the sand box. A sand layer of $200 \mathrm{~mm}$ is placed between two water layers on top and on bottom, separated from them by nonwoven fabrics and acrylic perforated layers. The water flow rate through the sand layer is kept constant by keeping constant the difference of water levels between the over flow pipe and the water outlet. A U-shaped line heater, connected to a voltage supply, is inserted into a stainless steel pipe $200 \mathrm{~mm}$ long with an external diameter of 3.2 $\mathrm{mm}$. The steel pipe is inserted horizontally into the vertical cylinder. Temperature is measured inside the steel pipe at the center and in the sand layer on a rectangular grid. All the equipment is placed into a thermostatic chamber. Reported Darcy velocities, obtained by flow rate measurements, range from $8.39 \cdot 10^{-6} \mathrm{~m} / \mathrm{s}$ to $6.22 \cdot 10^{-5} \mathrm{~m} / \mathrm{s}$. TRT injection rate is 6.6 $\mathrm{W} / \mathrm{m}$.

The set up by Wagner et al. [14] is a large tank (9 m $\times 6 \mathrm{~m} \times 4.5 \mathrm{~m}$ ) filled with 5 unconsolidated quartz layers with different granulometry. A constant hydraulic gradient can be established in the tank. The TRT is performed on a grouted borehole with a radius of $0.1 \mathrm{~m}$ and penetrating the upper $4.3 \mathrm{~m}$, equipped with a double $\mathrm{U}$ pipe. Measurements last for 8 days, including heating at $130 \mathrm{~W} / \mathrm{m}$ for the first 3 days and recovery for the subsequent 5 days. Darcy flow is derived from parameter estimation of the measured fluid temperature evolution and the corresponding hydraulic conductivity range is compared with estimates based on sieve analysis of the sand layers and literature correlations.

From the analysis of the mentioned laboratory set ups, the following considerations were derived:

- box sizes vary in a broad range, from a minimum of a few ten centimetres to a ten of meters. Unperturbed temperature conditions at the sand box boundaries are achieved either by conditioning the surrounding environment or by properly sizing the box.

- Most of the times the borehole and the U pipe are downscaled in depth but not in diameter, so that the height-to-diameter ratio is generally smaller in laboratory then in field.

- When the borehole and the U-pipe radius are not downscaled, heat injection is achieved as in real TRTs by controlling the carrier fluid temperature and flow rate; in turn, when the ground heat exchanger is significantly 
down-scaled, it is conveniently replaced by a heating cable.

- Groundwater flow can be simulated by establishing a proper hydraulic gradient through the medium; Darcy flow is either measured directly as the flow rate in the loop or inferred from the hydraulic properties of the sand.

- Physical scaling criteria are in general not explicit; only in [12] criteria for time scaling are reported.

Due to laboratory constraints, we decided to develop a small sand box apparatus with a significant geometrical down-scaling of the borehole and the medium. Physical scaling considerations, based on heat transfer similarity, were adopted since the design phase to determine geometrical, time and velocity scale factors. Miniaturization of the U-pipe led to simulate it by means of a heating cable. Perturbation of the medium boundaries due to heat injection was minimised by a proper sizing and checked during first experimental tests. A closed hydraulic loop was designed in order to vary the flow rate across the medium. The box was built in PMMA and structurally reinforced with wood panels, leaving a window for visual inspection of the medium.

\subsection{Physical scaling}

According to the MLS model, the dimensionless temperature increase in the medium $\Theta$ can be expressed as [6]:

$$
\Theta(R, \varphi, F o)=e^{\frac{R \cos \varphi}{2}} \int_{0}^{\left(R^{2} / 4 F o\right)} \frac{e^{\left(-\frac{1}{\eta}-\frac{R^{2} \eta}{16}\right)}}{2 \eta} d \eta
$$

where $R$ is the dimensionless radial coordinate, or local Péclet number, $\varphi$ is the polar angle and $F_{O}$ the dimensionless time. $R$ is defined as a function of the revised velocity $U$, the radial coordinate $r$ and the medium thermal diffusivity $\alpha$ as:

$$
\begin{aligned}
& R=\frac{U r}{\alpha} \\
& U=\frac{\rho_{w} c_{w} u}{\rho c}
\end{aligned}
$$

where $u$ is Darcy velocity, $\rho$ and $c$ represent the density and specific heat capacity of the medium (no subscript) or of the water (w subscript). Fourier number is defined as:

$$
F o=\frac{U^{2} t}{\alpha}
$$

where $t$ is time. At the laboratory scale, the borehole radius $r_{b h}$ ', the time $t^{\prime}$ and the velocity $U$ ' can be defined. The following scale factors $L_{r}, L_{t}$ and $L_{U}$ are then introduced as the ratios between the quantities at the laboratory scale and the corresponding quantities at the field scale:

$$
L_{r}=\frac{r_{b h^{\prime}}}{r_{b h}} \quad L_{t}=\frac{t \prime}{t} \quad L_{U}=\frac{U^{\prime}}{U}
$$

In order to conserve the relevant dimensionless number in equation (1) between the laboratory and the field scales, we have:

$$
\begin{gathered}
R^{\prime}=R \Rightarrow L_{U}=\frac{1}{L_{r}} \\
F O^{\prime}=F O \Rightarrow L_{U}{ }^{2}=\frac{1}{L_{t}}
\end{gathered}
$$

It was decided to down-scale the borehole radius by a factor 10, i.e. we set $L_{r}=10^{-1}$. Therefore equations (6) and (7) imply that $L_{t}=10^{-2}$ and $L_{U}=10$. Considering then a representative borehole radius $r_{b h}=12 \mathrm{~cm}$, a TRT duration of $72 \mathrm{~h}$, and a range of Darcy velocities in a sandy aquifer equal to $10^{-7} \mathrm{~m} / \mathrm{s}-10^{-5} \mathrm{~m} / \mathrm{s}$ [15], in the Sand Box apparatus the borehole radius becomes $r_{b h}$ ' $=$ $1.2 \mathrm{~cm}$, the TRT duration reduces to $0.72 \mathrm{~h}$ and the Darcy velocity range moves to $10^{-6} \mathrm{~m} / \mathrm{s}-10^{-4} \mathrm{~m} / \mathrm{s}$.

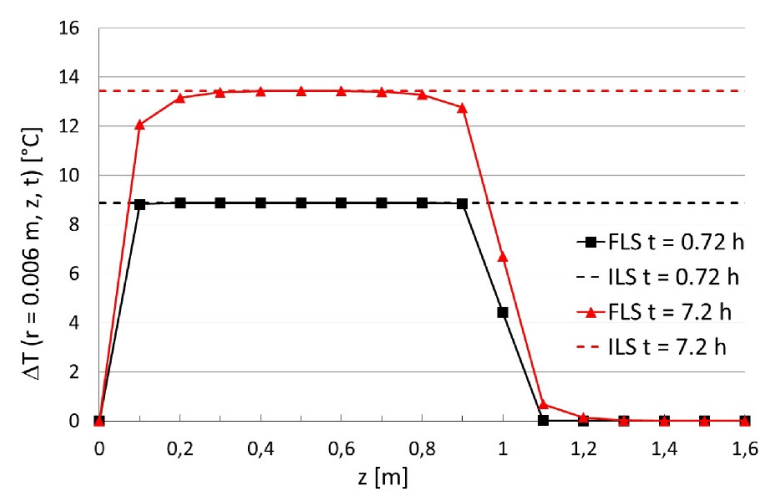

Fig. 1. Comparison between ILS and FLS temperature increase as a function of depth at radial distance $r=0.006 \mathrm{~m}$ for $t=0.72$ $\mathrm{h}$ and $t=7.2 \mathrm{~h}\left(q=50 \mathrm{~W} / \mathrm{m} ; \alpha=5.3 \cdot 10^{-7} \mathrm{~m}^{2} / \mathrm{s}\right)$

As far as the borehole depth at the laboratory scale is concerned, it was decided to set it to $H^{\prime}=1 \mathrm{~m}$. Considering a typical borehole depth in Italy equal to $H$ $=100 \mathrm{~m}$, the depth scaling factor is $L_{H}=H^{\prime} / H=10^{-2}$. This choice clearly resulted in a height-to-diameter ratio at the laboratory scale $10^{-1}$ times smaller than at the field scale, namely in a squatter heat source. The question then arised whether in the apparatus the effects of the finite dimension of the heat source would be more evident. According to [16] the axial effects tend to decrease as the Péclet number increases, thus the most critical condition corresponds to null groundwater 
velocity. Therefore we simulated the temperature variation produced in a medium with thermal diffusivity $\alpha=5.3 \cdot 10^{-7} \mathrm{~m}^{2} / \mathrm{s}$ by a finite line source with depth $H^{\prime}=$ $1 \mathrm{~m}$ and thermal injection $q=50 \mathrm{~W} / \mathrm{m}$ through the Finite Line Source (FLS) model [3]. We found that the temperature variation at the borehole radius for depths between $0.3 \mathrm{~m}$ and $0.7 \mathrm{~m}$ coincides with the ILS variation for times much larger than the TRT duration (Figure 1). Therefore, provided that temperature measurements are taken in such a range of depths, axial effects are negligible.

After sizing the borehole at the laboratory scale, the box sizes were identified, so that the distances between the borehole and the box borders guarantee that the thermal perturbation produced by the line source does not approach the borders until the TRT is over. The ILS model was used to identify the minimum distances from the upstream border (with respect to the groundwater direction) and from the side borders, leading to $L_{x, \text { upstream }}=L_{y} \geq 10 \mathrm{~cm}$. The MLS model at the maximum expected Darcy velocity $\left(u_{\max }=10^{-4} \mathrm{~m} / \mathrm{s}\right)$ was used to identify the minimum distance from the downstream border, resulting in $L_{x, \text { downstream }} \geq 48 \mathrm{~cm}$. Finally the FLS model allowed to identify the minimum distance from the bottom of the source, giving $L_{z} \geq 7$ $\mathrm{cm}$.

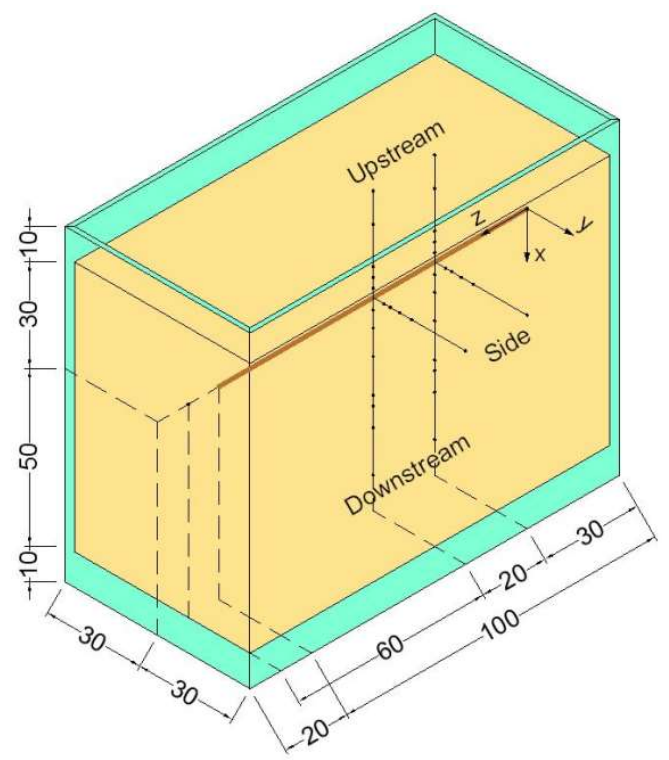

Fig. 2. Sand Box apparatus and borehole (sizes in $\mathrm{cm}$ ). The position of some temperature probes is also shown.

The final Sand Box sizes $(60 \mathrm{~cm} \times 120 \mathrm{~cm} \times 100 \mathrm{~cm})$ are shown in Figure 2. The overall height is $100 \mathrm{~cm}$, being composed of $10 \mathrm{~cm}$ for the upper water layer, 80 $\mathrm{cm}$ of porous medium and $10 \mathrm{~cm}$ for the lower water layer. The box is $120 \mathrm{~cm}$ along the borehole depth and $60 \mathrm{~cm}$ wide. The borehole is placed horizontally at 30 $\mathrm{cm}$ from the top surface of the medium, at $50 \mathrm{~cm}$ from the bottom surface and at $30 \mathrm{~cm}$ from both side borders.

\subsection{Hydraulic loop design}

The hydraulic loop was designed considering that, as stated in subsection 2.2, the Darcy velocity had to span the range $10^{-6} \mathrm{~m} / \mathrm{s}-10^{-4} \mathrm{~m} / \mathrm{s}$. Therefore, the water flow rate had to vary within $2.592 \mathrm{dm}^{3} / \mathrm{h}$ and $259.2 \mathrm{dm}^{3} / \mathrm{h}$, which is a two orders of magnitude span. The pump chosen for this purpose is a peristaltic pump (Cheimika $\mathrm{BT} / 600 / \mathrm{S}$ ) in which the delivered water flow rate variation is achieved changing the pump RPM (range 0,1 RPM - 600 RPM) and the diameter of the flexible tube the pump is equipped with (two tubes, $4.8 \mathrm{~mm}$ and 9.6 $\mathrm{mm})$.

Furthermore, two requests of flow rate uniformity over cross section area and stability over time during experiments arose in the design of the hydraulic loop. To this goal, a set of two consecutive strainers was used both in the water inlet and outlet sections. In the inlet section, the first flow strainer is a pre-drilled metallic slab and the second strainer is a light weight coarse filter whereas in the outlet sections the two layers are switched.

The hydraulic loop is then completed introducing a $\mathrm{Y}$ strainer, for sand removal, and two ball valves that are closed only during filter cleaning. It is worth specifying that the peristaltic pump may work with dirty fluid, i.e. water + sand, but the $\mathrm{Y}$ strainer is needed for safe operation of the water flow rate measurement device.

A schematic of the final hydraulic loop is shown in Figure 3.

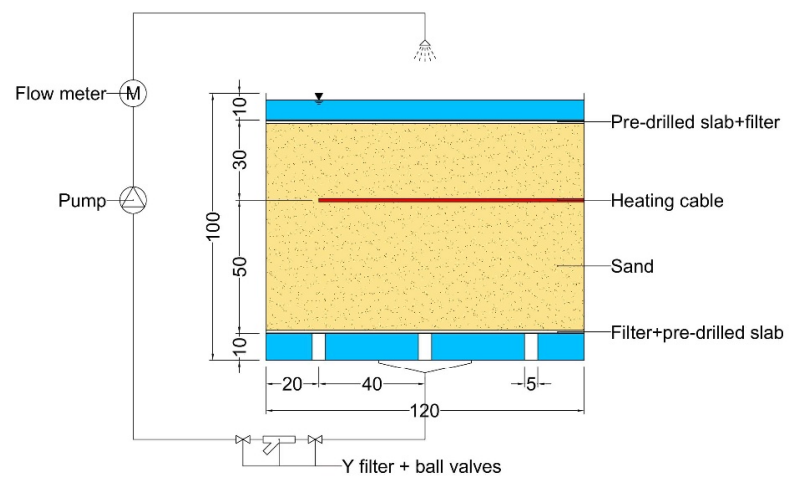

Fig. 3. Hydraulic loop layout and Sand Box side view (dimensions in $\mathrm{cm}$ )

Finally, two different flow rate sensors were considered in the hydraulic loop. This choice came from the abovementioned range of Darcy velocity considered in the hydraulic loop design. The measurement device 
chosen for the range of low flow rate is a gear flow rate sensor (KEM, ZHM 01 series) that can measure the water flow rate in the range $0.12 \mathrm{dm}^{3} / \mathrm{h}-30 \mathrm{dm}^{3} / \mathrm{h}$ with a maximum uncertainty equal to $\pm 1 \%$. Conversely, for the range of high flow rate a vortex flow rate sensor (Huba Control, 210 series) that can measure the water flow rate in the range $30 \mathrm{dm}^{3} / \mathrm{h}-600 \mathrm{dm}^{3} / \mathrm{h}$ with an uncertainty equal to $\pm 1 \%$ r.v. (flow lower than $50 \%$ f.s.) and $\pm 2 \%$ r.v. (flow higher than $50 \%$ f.s.) is used.

\subsection{Heat source}

Due to the important down-scaling of the borehole, for the sake of simplicity the U-pipe was replaced by two heating wires (diameter $3 \mathrm{~mm}$, length $1 \mathrm{~m}$, electrical resistance $5.38 \Omega$ ). The minimum bending radius of the wires is not compatible with the borehole diameter of 12 $\mathrm{mm}$ so that the bottom part of the $\mathrm{U}$ is not reproduced. The wires are connected in parallel to a constant voltage supply in order to dissipate $25 \mathrm{~W} / \mathrm{m}$ each, or $50 \mathrm{~W} / \mathrm{m}$ as a whole. Clearly, by setting a different voltage, the heat injection rate can be easily varied. The wires were inserted into a copper tube representing the borehole wall and the tube was backfilled with the high thermal conductivity grout Termoplast Plus®.

\subsection{Soil}

Fine silica sand from Po river was chosen to fill the Sand Box. From the granulometric curve the median grain diameter was identified as $\mathrm{d}_{50}=0.275 \mathrm{~mm}$ and the uniformity coefficient $d_{60} / d_{10}=2$. By means of Prugh charts the coefficient of permeability of the sand was then estimated as $k=3 \cdot 10^{-4} \mathrm{~m} / \mathrm{s}$. Porosity was measured by comparing the mass of the sand sample as is and the mass of the water saturated sample, obtaining $\phi=0.374$ \pm 0.003 .

By knowing the porosity, the volume of water required to saturate the desired sand volume was firstly estimated. The water volume was then inserted into the Sand Box and a raining technique was adopted in order to insert the proper quantity of sand into the water, similar to [12].

\subsection{Measurement system}

Measurements are performed by means of a data acquisition system National Instruments cDAQ-9178 equipped with 4 NI-9214 modules for temperature measurements with thermocouples and 1 NI-9207 module for voltage and current measurements. The acquisition is remotely controlled by a LabView programme.

50 needle T-type thermocouples (diameter $1 \mathrm{~mm}$, length $150 \mathrm{~mm}$ ) were inserted into the medium on two planes, namely at $\mathrm{z}=30 \mathrm{~cm}$ and $\mathrm{z}=50 \mathrm{~cm}$. In each plane, the probes were distributed on a vertical line (upstream and downstream of the borehole) and on a horizontal line (at the borehole side), as shown in Figure 2. After setting the minimum distance between adjacent probes at $1 \mathrm{~cm}$, the probes distribution was chosen on the basis of the expected temperature profile, resulting in a denser arrangement close to the heat source. In each plane 3 temperature probes were positioned at the borehole wall at $\theta=0^{\circ}, 90^{\circ}, 180^{\circ}$ with respect to $\mathrm{x}$ axis. Such probes are used to derive an average borehole wall temperature $T_{b h}$ to be directly interpolated with either the ILS or the MLS model. Some further probes are positioned at the medium borders, to control the boundary condition, and another probe is inserted into the hydraulic loop to measure the inlet water temperature. All the thermocouples with the relative measurement chain were calibrated against a reference thermometer, achieving a calibration accuracy of $\pm 0.1^{\circ} \mathrm{C}$.

As already mentioned in subsection 2.3 , the flow rate in the hydraulic loop is measured by means of a flowmeter, connected to the voltage/current module.

\section{Experimental tests and results}

The high velocity hydraulic loop was firstly tested, by equipping the peristaltic pump with the large diameter tube and by mounting the vortex flow rate sensor. If the pump rotor velocity is varied in the range $200 \div 600$ RPM, the Darcy velocity is found in the range $2.45 \cdot 10^{-5}$ $\div 7.46 \cdot 10^{-5} \mathrm{~m} / \mathrm{s}$. The maximum velocity achieved is thus in agreement with the design maximum value of $10^{-4} \mathrm{~m} / \mathrm{s}$ (subsection 2.2).

Before starting any TRT the temperature uniformity in the soil is checked and established. To this purpose, for the TRTs including groundwater flow, the desired water flow rate is forced across the sand for the necessary time before switching on the heat source. The TRT minimum duration is set to $0,72 \mathrm{~h}(2592 \mathrm{~s})$ and the time sampling to $1 \mathrm{~s}$.

In the following, the results of the TRT at null groundwater velocity and at the maximum velocity are reported. 


\subsection{TRT at null velocity}

First of all, a set of TRTs at null groundwater velocity was carried out, in order to test the apparatus in the simplest condition and to derive a reference thermal conductivity of the medium. As shown in Table 1, test 1 and 2 were performed with the same heat rate of about $50 \mathrm{~W} / \mathrm{m}$, while in test 3 a lower heat rate equal to 15.5 $\mathrm{W} / \mathrm{m}$ was adopted.

Table 1. TRTs at null velocity: specific heat rate and measured thermal conductivity at $\mathrm{z}=50 \mathrm{~cm}$ and $\mathrm{z}=30 \mathrm{~cm}$

\begin{tabular}{|c|r|r|r|}
\hline Test & $\begin{array}{c}\dot{\boldsymbol{q}} \\
{[\mathbf{W} / \mathbf{m}]}\end{array}$ & $\begin{array}{c}\lambda_{\mathbf{5 0}} \\
{[\mathbf{W} /(\mathbf{m} . \mathbf{K})]}\end{array}$ & $\begin{array}{c}\lambda_{\mathbf{3 0}} \\
{[\mathbf{W} /(\mathbf{m} . \mathbf{K})]}\end{array}$ \\
\hline 1 & 49.9 & $1.880 \pm 0.009$ & $1.875 \pm 0.009$ \\
\hline 2 & 50.2 & $1.972 \pm 0.018$ & $1.941 \pm 0.016$ \\
\hline 3 & 15.5 & $1.855 \pm 0.015$ & $1.843 \pm 0.014$ \\
\hline
\end{tabular}

As an example, in Figure 4 the temperature evolution with time measured at $\mathrm{z}=50 \mathrm{~cm}$ in the different positions $y$ at the borehole side $\left(\theta=90^{\circ}\right)$ during test 2 is shown. At $0,72 \mathrm{~h}$ from the heat source switch on the temperature increase at the borehole wall $(\mathrm{y}=0.6 \mathrm{~cm})$ is about $8^{\circ} \mathrm{C}$. At the Sand Box border $(\mathrm{y}=30 \mathrm{~cm})$ the temperature is stable even after about $2 \mathrm{~h}$ from the TRT beginning, proving that the Sand Box size along the $y$ axis is adequate. The time evolution of the average borehole wall temperature at $\mathrm{z}=50 \mathrm{~cm}$ and at $\mathrm{z}=30 \mathrm{~cm}$ was analysed by means of the ILS model as in the standard TRT approach [4, 17], i.e. by interpolating with the following curve:

$$
T_{b h}(t)=A \ln (t)+B \quad A=\frac{\dot{q}}{4 \pi \lambda}
$$

The analysis provided a measure of the soil thermal conductivity, reported in Table 1 as $\lambda_{50}$ and $\lambda_{30}$ respectively. It can be noticed that for each test $\lambda_{50}$ and $\lambda_{30}$ are equal within the measurement accuracy. In turn, thermal conductivities derived from different tests differ from each other by more than the uncertainties. However, the standard deviation between them is only $2.4 \%$ of the average value, so that the outcomes of the tests can be considered in good agreement. Then the average thermal conductivity of the sandy soil is found to be $\lambda=(1.894 \pm 0.026) \mathrm{W} /(\mathrm{m} \cdot \mathrm{K})$, a value compatible with literature ranges for this kind of ground [17].

\subsection{TRT at maximum velocity}

The Sand Box apparatus was then tested near the upper limit of the velocity range, by performing a TRT with an average heat rate input of $50.3 \mathrm{~W} / \mathrm{m}$. At $580 \mathrm{RPM}$ the Darcy velocity across the medium is found to be $u=6.75$ $\cdot 10^{-5} \mathrm{~m} / \mathrm{s}$.

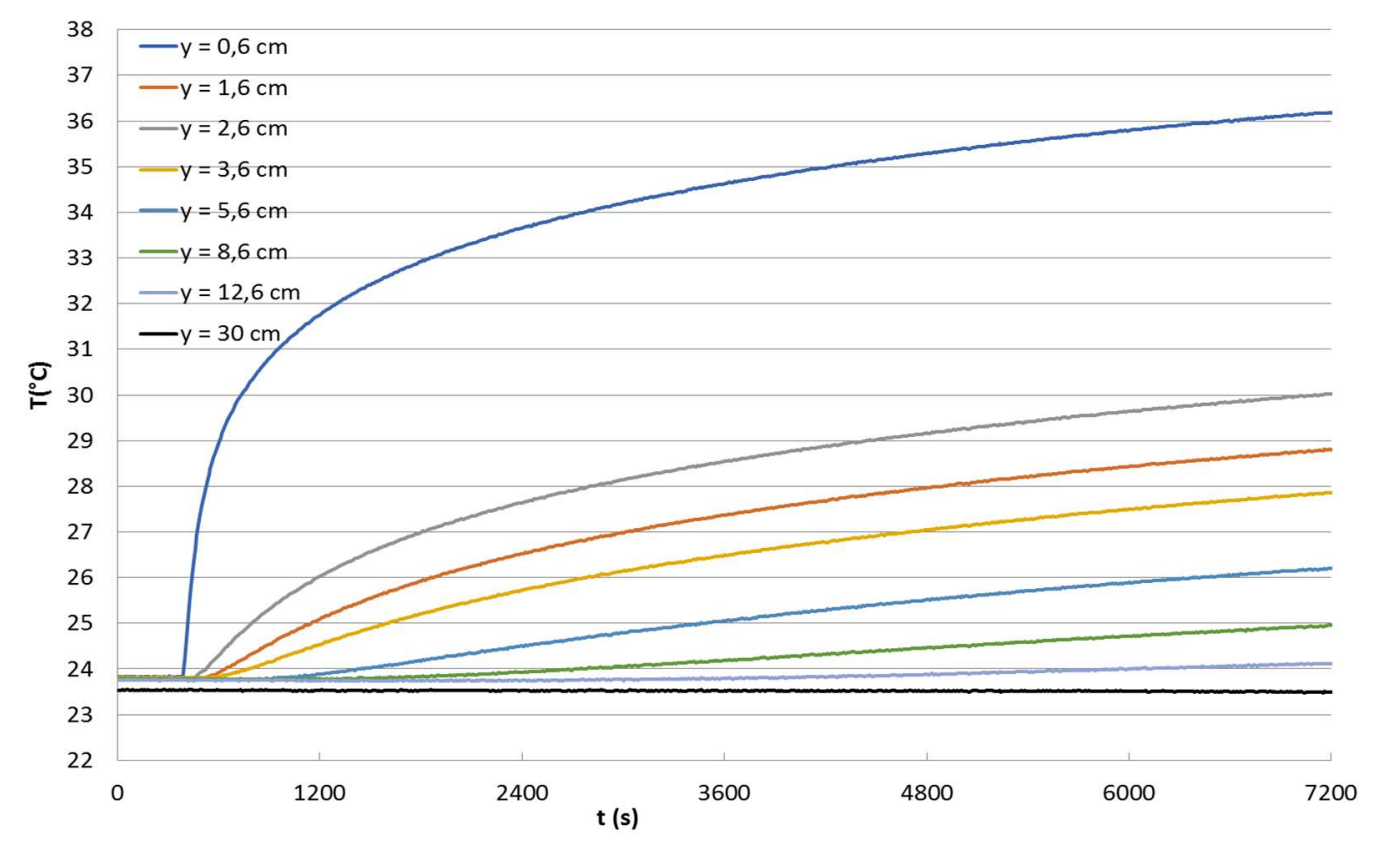

Fig. 4. TRT at null velocity $(\dot{q}=50.2 \mathrm{~W} / \mathrm{m})$ : temperature variation with time at $\mathrm{z}=50 \mathrm{~cm}$ for different coordinates y (borehole side) 


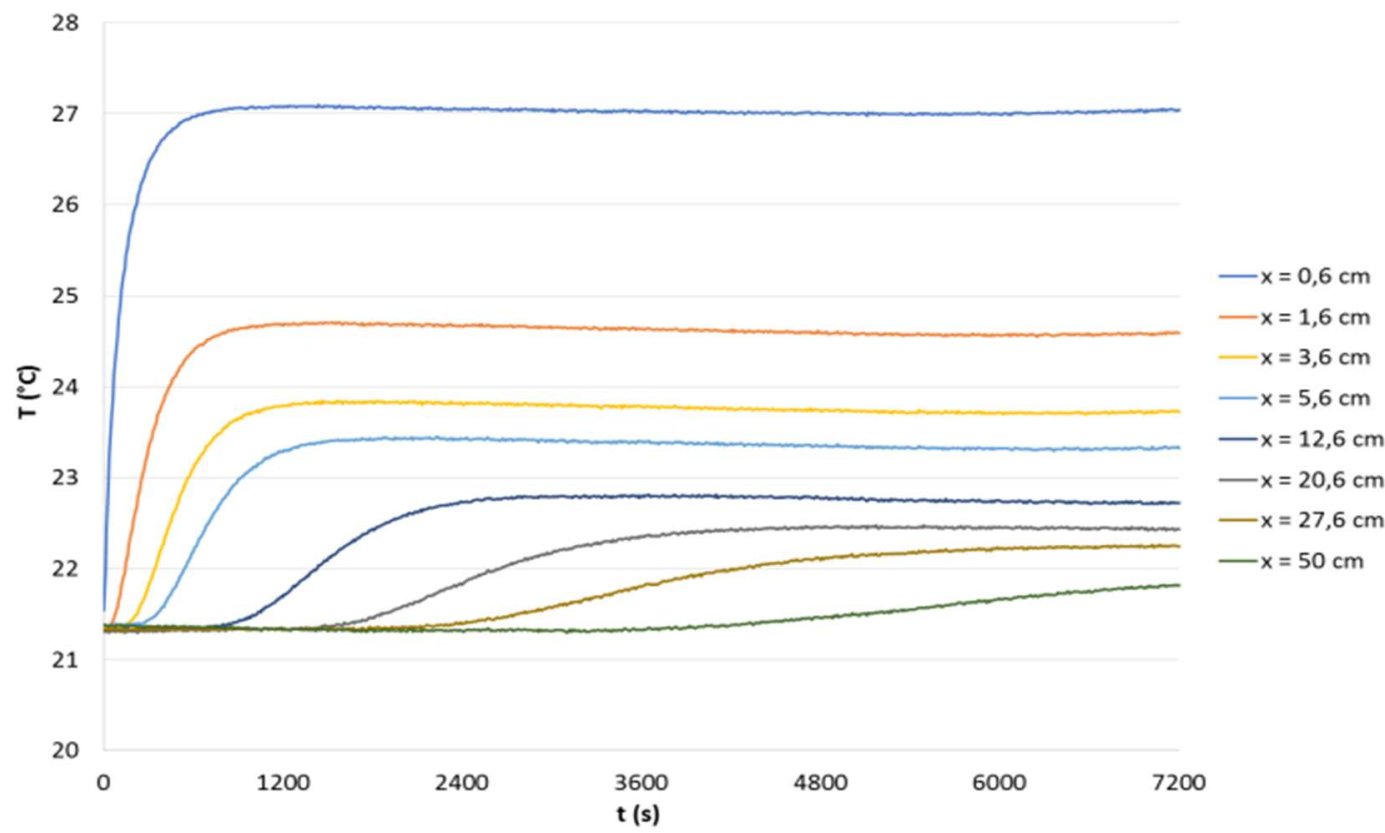

Fig. 5. TRT at $u=6.75 \cdot 10^{-5} \mathrm{~m} / \mathrm{s} \quad(\dot{q}=50.3 \mathrm{~W} / \mathrm{m})$ : temperature variation with time at $\mathrm{z}=50 \mathrm{~cm}$ for different coordinates $\mathrm{x}$ (downstream i.e. $\theta=0^{\circ}$ )

Figure 5 shows the soil temperature increase with time at $\mathrm{z}=50 \mathrm{~cm}$ in some probes located downstream, the first being at the borehole wall $(\mathrm{x}=0,6 \mathrm{~cm})$ and the last one at the sand bottom $(\mathrm{x}=50 \mathrm{~cm})$. At $0.72 \mathrm{~h}$ from the heat source switch on the borehole wall temperature rise is about $6^{\circ} \mathrm{C}$ and, moreover, steady state has already been reached approximately after $1200 \mathrm{~s}$.

The farther is the distance $\mathrm{x}$ from the line source, the smaller is the temperature increase and the longer the time to reach steady state. The temperature at the sand bottom remains unperturbed for at least $1 \mathrm{~h}$ and after $2 \mathrm{~h}$ the temperature rise is only $0.8^{\circ} \mathrm{C}$. Therefore the Sand Box size in the downstream direction is suitable for performing a TRT lasting $0.72 \mathrm{~h}$ under groundwater flow up to this velocity.

Figure 6 shows the temperature variation with the $\mathrm{x}$ coordinate on the plane containing the borehole $(y=0)$ at different times. It is worth to remind that upstream and

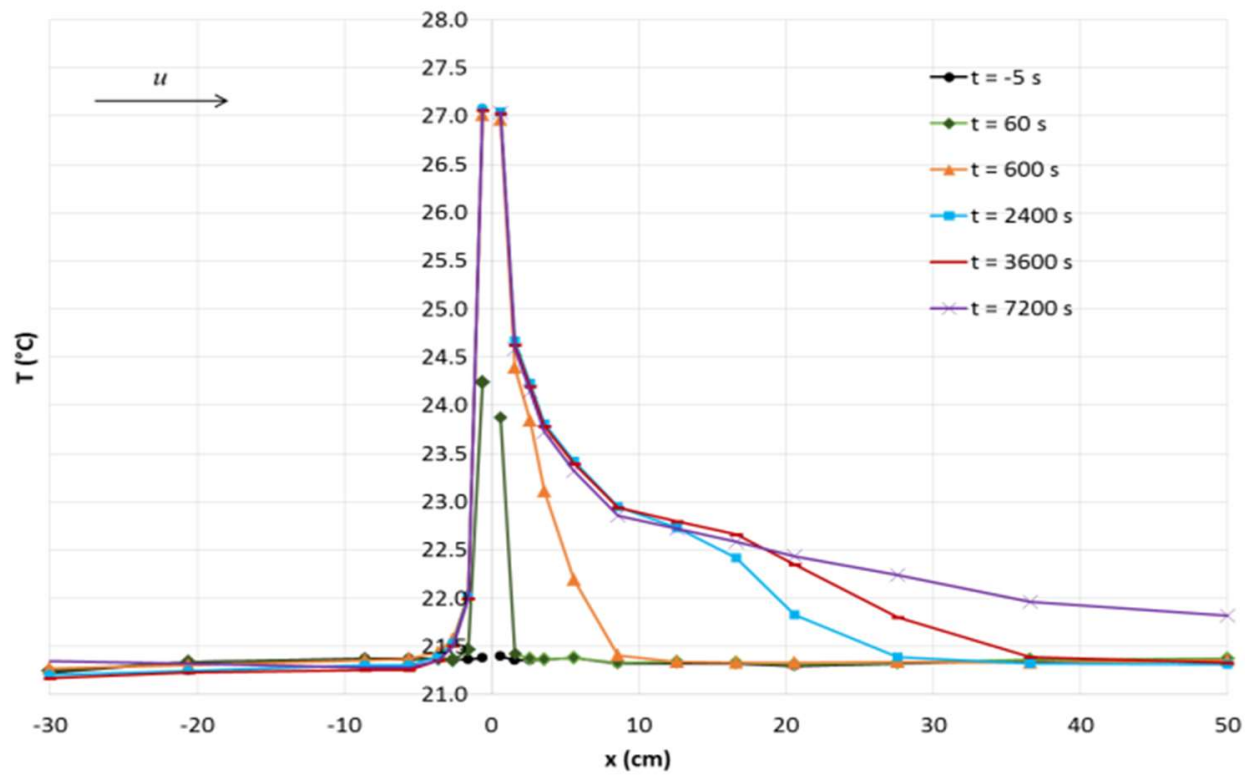

Fig. 6. TRT at $u=6.75 \cdot 10^{-5} \mathrm{~m} / \mathrm{s}(\dot{q}=50.3 \mathrm{~W} / \mathrm{m})$ : temperature profile along $\mathrm{x}$ in $\mathrm{y}=0, \mathrm{z}=50 \mathrm{~cm}$ at different times 
downstream positions correspond to negative and positive values of $\mathrm{x}$ respectively. The characteristic asymmetric profile predicted by the MLS model can be clearly noticed since the first $600 \mathrm{~s}$.

However, a careful inspection of the temperatures measured by the 2 probes at the borehole wall is needed. Before the heat source is switched on $(t=-5 \mathrm{~s})$, the temperature in $\mathrm{x}=-0.6 \mathrm{~cm}$ and in $\mathrm{x}=+0.6 \mathrm{~cm}$ is the same within the measurement accuracy. Then, at $t=60$ $\mathrm{s}$, the upstream temperature is about $0.5^{\circ} \mathrm{C}$ higher than the downstream and from $\mathrm{t}=600 \mathrm{~s}$ on they become equal again. This overall behaviour is not coherent with the MLS prediction, since at equal distance from the heat source the upstream temperature is expected to be lower than the downstream. The observed behaviour may be caused by the location of the two wires inside the borehole that simulate the branches of the $U$ pipe. Actually the effective position of the wires was not controlled when the borehole was filled with grout and thus it is possible that both of them are located in the upper part of the borehole section. This consideration is supported also by the inspection of the same temperatures during the null velocity TRT.

\section{Conclusions and prospects}

The Sand Box apparatus developed in this research allows to perform TRT under groundwater flow in the Darcy velocity range from zero up to about $7 \cdot 10^{-5} \mathrm{~m} / \mathrm{s}$ at the laboratory scale, corresponding to $7 \cdot 10^{-6} \mathrm{~m} / \mathrm{s}$ at the field scale. Preliminary tests executed at null velocity and at the maximum velocity prove that the Sand Box boundaries are not affected by the heat source for the typical TRT duration and thus that the apparatus is properly sized. The ground conductivity derived from TRT at null velocity and LS interpolation is equal to $\lambda=$ $(1.894 \pm 0.026) \mathrm{W} /(\mathrm{m} \cdot \mathrm{K})$, which is coherent with the literature range for a saturated sand. As a next step, the thermal conductivity of a sample of saturated sand will be measured through another technique, in order to provide an independent validation of the Sand Box apparatus and methodology.

The first test performed with groundwater flow shows the development of the characteristic asymmetric temperature profile around the borehole and the achievement of a steady state regime. At the same time the positioning of the wires, representing the U-pipe, inside the borehole is critical since a local measure is performed at the borehole wall.

The next step will consist in executing TRT at different Darcy velocities and the corresponding analysis with the MLS model, in order to derive both thermal conductivity and Darcy velocity and to investigate the suitability of this approach to interpret the test.

\section{References}

1. S. Gehlin, J. Spitler, Renewable and Sustainable Energy Reviews, 50 (2015)

2. C. Zhang, Z. Guo, Y. Liu, X. Cong, D. Peng, Renewable and Sustainable Energy Reviews, 40 (2014)

3. H.S. Carslaw, J.C. Jaeger, Conduction of heat in solids (Oxford: Clarendon Press, 1959)

4. EN ISO 17628 (2015)

5. Witte HJL, Proc. Worskhop Europeen sur Test de Reponse Geothermique (Lausanne, $\mathrm{CH}, 2001$ )

6. Diao N, Li Q, Fang Z, International Journal of Thermal Sciences, 43 (2004)

7. Wagner V, Blum $P$, Kübert $M$, Bayer $P$, Geothermics, 46 (2013)

8. A. Chiasson, A. O'Connell, HVAC\&R Research, 17, 6 (2011)

9. A. Angelotti, F. Ly, A. Zille, Geothermal Energy 6, 12 (2018)

10. J. Rouleau, L. Gosselin, International Journal of Thermal Sciences, 109 (2016)

11. R.A. Beier, M.D. Smith, J.D. Spitler, Geothermics, 40 (2011)

12. C.A. Kramer, O. Ghasemi-Fare, P. Basu, Geotechnical and Geological Engineering, 33, 2 (2014)

13. T. Katsura, K. Nagano, S. Takeda, K. Shimakura, Proc. Ecostock (Stockton, USA, 2006)

14. V. Wagner, P. Bayer, G. Bisch, M. Kubert, P. Blum, Water Resources Research, 50 (2014)

15. A. Angelotti, L. Alberti, I. La Licata, M. Antelmi, Energy Conversion and Management, 77 (2014)

16. N. Molina-Giraldo, P. Blum, K. Zhu, P. Bayer, Z. Fang, International Journal of Thermal Sciences, 50 (2011)

17. UNI 11466 (2011) 